\title{
Innovations in Radiotherapy Planning of Head and Neck Cancers: Role of PET
}

Esther G.C. Troost ${ }^{1}$, Dominic A.X. Schinagl ${ }^{1}$, Johan Bussink ${ }^{1}$, Otto C. Boerman ${ }^{2}$, Albert J. van der Kogel ${ }^{1}$, Wim J.G. Oyen ${ }^{2}$, and Johannes H.A.M. Kaanders ${ }^{1}$

${ }^{1}$ Department of Radiation Oncology, Radboud University Nijmegen Medical Centre, Nijmegen, The Netherlands; and ${ }^{2}$ Department of Nuclear Medicine, Radboud University Nijmegen Medical Centre, Nijmegen, The Netherlands

Learning Objectives: On successful completion of this activity, participants should be able to describe (1) the role of ${ }^{18} \mathrm{~F}-\mathrm{FDG}$ PET for target volume delineation in radiation treatment and unresolved issues; (2) the relevance of biologic PET tracers for noninvasive tumor characterization, possibly enabling patient selection and treatment monitoring; and (3) the possibilities for incorporating PET information into radiotherapy planning and into adaptation of the irradiated volume during treatment.

Financial Disclosure: The authors of this article have indicated no relevant relationships that could be perceived as a real or apparent conflict of interest.

CME Credit: SNM is accredited by the Accreditation Council for Continuing Medical Education (ACCME) to sponsor continuing education for physicians. SNM designates each JNM continuing education article for a maximum of 1.0 AMA PRA Category 1 Credit. Physicians should claim only credit commensurate with the extent of their participation in the activity.

For CE credit, participants can access this activity through the SNM Web site (http://www.snm.org/ce_online) through January 2011.

Modern radiotherapy techniques heavily rely on high-quality medical imaging. PET provides biologic information about the tumor, complementary to anatomic imaging. Integrated PET/ $\mathrm{CT}$ has found its way into the practice of radiation oncology, and ${ }^{18} \mathrm{~F}-\mathrm{FDG}$ PET is being introduced for radiotherapy planning. The functional information possibly augments accurate delineation and treatment of the tumor and its extensions while reducing the dose to surrounding healthy tissues. In addition to ${ }^{18} \mathrm{~F}-\mathrm{FDG}$, other PET tracers are available for imaging specific biologic tumor characteristics determining radiation resistance. For head and neck cancer, the potential gains of PET are increasingly being recognized. This review describes the current role of PET and perspectives on its future use for selection and delineation of radiotherapy target volumes and for biologic characterization of this tumor entity. Furthermore, the potential role of PET for early response monitoring, treatment modification, and patient selection is addressed in this review.

Key Words: ${ }^{18}$ F-FDG PET; head and neck carcinoma; radiotherapy planning; hypoxia; proliferation; radioresistance

J Nucl Med 2010; 51:66-76

DOI: 10.2967/jnumed.108.061499

$\mathbf{T}$ he field of radiation oncology changed dramatically with the wide introduction of computer-optimized intensity-modulated radiation therapy (IMRT) in the 1990s.

Received Dec. 22, 2008; revision accepted Mar. 25, 2009.

For correspondence or reprints contact: Esther G.C. Troost, Department of Radiation Oncology, Radboud University Nijmegen

Medical Centre, P.O. Box 9101, 6500 HB Nijmegen, The Netherlands.

E-mail: e.troost@rther.umcn.n

COPYRIGHT @ 2010 by the Society of Nuclear Medicine, Inc.
IMRT is based on the use of numerous radiation beams with optimized nonuniform intensities resulting from inverse treatment planning. The algorithm for beam fluence calculations is guided by dose-volume objectives for the target volume and organs at risk delineated by the radiation oncologist. IMRT can thus achieve much better dose conformity than conventional radiotherapy techniques. With this technique, different dose prescriptions to multiple target sites can be delivered. It also facilitates boosting of high radiation doses to the primary tumor while reducing the dose to radiation-sensitive tissues adjacent to the tumor $(1,2)$. Because of the highly conformal dose distribution and steep dose gradients used in IMRT, knowledge about the localization and boundaries of the primary tumor and of the cervical lymph node metastases is crucial. For this purpose, biologic imaging using PET may augment traditional imaging methods such as CT and MRI (Fig. 1).

\section{PET FOR HIGHLY ACCURATE RADIATION TREATMENT PLANNING}

\section{Identification of Radiotherapy Targets Based on ${ }^{18}$ F-FDG PET}

In a recent issue of this journal, Fletcher et al. reviewed the available literature, and a multidisciplinary expert panel developed recommendations on the use of ${ }^{18} \mathrm{~F}$-FDG PET in oncology practice (3). These recommendations on the use of ${ }^{18}$ F-FDG PET for the detection and staging of head and neck tumors are briefly summarized here.

The expert panel concluded that ${ }^{18}$ F-FDG PET should not be added to conventional anatomic imaging in the 


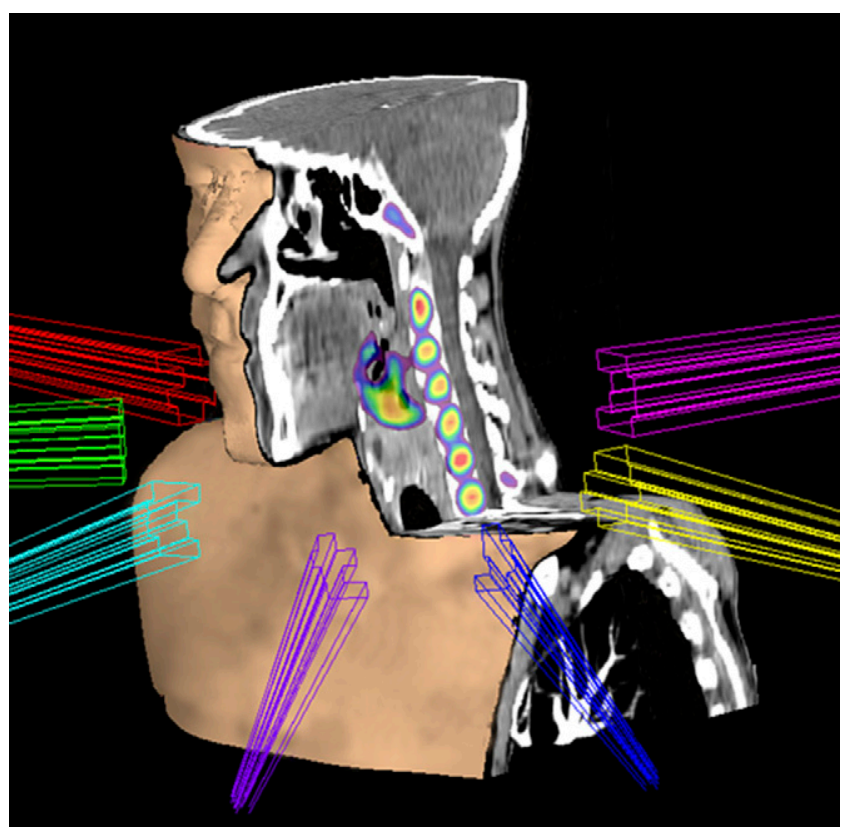

FIGURE 1. ${ }^{18} \mathrm{~F}-\mathrm{FLT}$ PET/CT scan for image-guided highprecision radiation treatment planning in oropharynx carcinoma.

routine diagnostic work-up of primary head and neck tumors (3). This conclusion was drawn because the available data were too uncertain as to whether ${ }^{18} \mathrm{~F}$-FDG PET can determine the anatomic extent of the primary tumor more accurately than CT or MRI.

Regarding the detection of cervical lymph node metastases, Fletcher et al. concluded that ${ }^{18}$ F-FDG PET has a higher sensitivity, specificity, positive predictive value, and negative predictive value than CT and MRI. Therefore, its use in routine local staging was recommended (3). However, this recommendation did not incorporate the findings of the metaanalysis by Kyzas et al. that was published shortly thereafter (4). This metaanalysis reviewed 35 studies using ${ }^{18}$ F-FDG PET for the pretreatment evaluation of the lymph node status. The authors concluded that there was no solid evidence to support the routine application of ${ }^{18}$ F-FDG PET, as the sensitivity and specificity improved by only 5\%-7\% compared with conventional imaging modalities. In the subset of studies only enrolling patients without clinically apparent cervical lymph node metastases, the sensitivity was only $50 \%$ and not better than conventional imaging methods, specifically ultrasound with fine-needle cytology. From these contradictory recommendations, it is clear that this is an unresolved issue that requires further study.

For the detection of distant metastases, the net benefit of using ${ }^{18}$ F-FDG PET was reported to be still uncertain (3). Functional imaging might be beneficial in patients with advanced-stage disease, in whom the odds of having distant metastases are greater (3). In these patients, the ${ }^{18} \mathrm{~F}-\mathrm{FDG}$ PET findings may alter the treatment intention from curative to palliative and thus affect the total dose and fractionation scheme. Additionally, it may reduce treatment-related side effects in those patients, as the selected treatment volume is often confined to the primary tumor or metastatic lymph nodes causing discomfort or pain.

The role of ${ }^{18} \mathrm{~F}$-FDG PET or ${ }^{18} \mathrm{~F}$-FDG PET/CT for the early detection of recurrent disease is addressed elsewhere $(3,5,6)$.

It can be concluded that there is only a modest role for ${ }^{18} \mathrm{~F}$-FDG PET in the routine diagnostic workup and staging of patients with head and neck cancer. However, this does not disqualify ${ }^{18} \mathrm{~F}-\mathrm{FDG}$ PET as a potentially useful and complementary tool for accurate delineation of the radiotherapy target volume and customized dose delivery.

\section{${ }^{18}$ F-FDG PET for Delineation of Radiotherapy Target Volume}

In this era of high-precision radiotherapy, accurate tumor volume delineation with respect to tumor boundaries, shape, and volume is crucial. Target volume delineation is primarily based on anatomic information about the tumor and affected lymph nodes. A thorough physical examination of the head and neck forms the basis for assessment of tumor extension, especially for superficially spreading mucosal tumors. Anatomic imaging using CT or MRI provides important complementary information by depicting distorted anatomy and regions of abnormal contrast enhancement. For oral cavity and oropharyngeal carcinomas, MRI is the preferred imaging modality because it achieves better soft-tissue contrast.

There are several potential advantages of using ${ }^{18} \mathrm{~F}-\mathrm{FDG}$ PET for target volume delineation. ${ }^{18}$ F-FDG PET may reduce the interobserver variability in gross tumor volume (GTV) delineation, reduce the size of the GTV, identify tumor areas or lymph nodes missed by CT or MRI, and identify parts of the GTV potentially requiring an additional radiation dose. However, the use of ${ }^{18}$ F-FDG PET also bears some disadvantages: the limited spatial resolution, the lack of a standardized method for signal segmentation, and falsepositive ${ }^{18}$ F-FDG PET readings caused by inflammation.

A reduction of interobserver variability has been demonstrated for non-small cell lung cancer when ${ }^{18} \mathrm{~F}-\mathrm{FDG}$ PET was incorporated in GTV delineation $(7,8)$. In patients with head and neck cancer, this finding has been less consistent. Ciernik et al. investigated the value of ${ }^{18} \mathrm{~F}-\mathrm{FDG}$ PET in 39 patients with various solid tumors, of which 12 were head and neck cancer (9). The investigators found both increases $(\geq 25 \%)$ and decreases $(\leq 25 \%)$ in half the patients when GTV delineation based on CT alone was compared with ${ }^{18}$ F-FDG PET/CT. When GTV delineation was compared between 2 experienced radiation oncologists, the mean volume difference of $26.6 \mathrm{~cm}^{3}$ by $\mathrm{CT}$ alone was reduced to $9.1 \mathrm{~cm}^{3}$ with ${ }^{18} \mathrm{~F}-\mathrm{FDG}$ PET/CT (9). Riegel et al. found conflicting results when 2 experienced radiation oncologists and 2 neuroradiologists delineated 16 patients with head and neck cancer (10). On average, the GTVs 
based on ${ }^{18}$ F-FDG PET/CT were larger than the corresponding CT-based volumes. Furthermore, the authors observed a large discrepancy between the GTV delineation of the 2 radiation oncologists, with one delineating larger volumes on CT and the other on ${ }^{18} \mathrm{~F}$-FDG PET/CT (10). One important difference between these 2 studies relates to thresholding of the ${ }^{18} \mathrm{~F}-\mathrm{FDG}$ PET signal: Ciernik et al. chose a fixed threshold of $50 \%$ of the maximum signal intensity, whereas Riegel et al. used a discretionary windowlevel setting $(9,10)$.

A reduction of the GTV using ${ }^{18}$ F-FDG PET has been demonstrated in a landmark study including laryngeal cancer patients (11). The authors investigated the role of coregistered CT, MRI, and ${ }^{18}$ F-FDG PET in GTV delineation of patients undergoing laryngectomy. Compared with the reference surgical specimen, ${ }^{18} \mathrm{~F}-\mathrm{FDG}$ PET came closest to depicting the true tumor volume. All modalities overestimated the extent of the tumor, ${ }^{18} \mathrm{~F}$-FDG PET by an average of $29 \%$, CT by $65 \%$, and MRI by $89 \%$. However, all 3 imaging modalities, including ${ }^{18} \mathrm{~F}-\mathrm{FDG}$ PET, failed to identify a small fraction of the macroscopic tumor (approximately $10 \%$ ), mainly consisting of superficial mucosal extensions.

Before PET-based GTVs can reliably and reproducibly be incorporated into high-precision radiotherapy planning, operator-independent segmentation tools have to be developed and validated. Simple visual interpretation of the PET signal is most commonly applied but is highly operator-dependent, as it is susceptible to the window-level settings of the images and interpretation differences $(10,12,13)$. This is why research groups have explored more objective methods, such as isocontouring based on a fixed standardized uptake value (SUV), for example, of 2.5 , or thresholds acquired through phantom experiments such as a fixed threshold of the maximum tumor signal intensity $(40 \%$ or $50 \%)(9,14,15)$. Daisne et al. used a variable threshold adaptive to the signal-to-background ratio in their study on laryngeal cancer patients (16). Recently, the same group published a new gradient-based segmentation tool based on watershed transform and hierarchical cluster analysis and validated this in an adaptive biologic image-guided planning study $(17,18)$. Shortly thereafter, van Dalen et al. published an iterative background-subtracted relative-threshold level method validated in patients with liver metastases (19). The optimal relative-threshold level thereby depends on the lesion size but not on the signal-to-background ratio.

A recent study on 78 patients with head and neck cancer compared 5 commonly used methods of ${ }^{18} \mathrm{~F}-\mathrm{FDG}$ PET signal segmentation (visual interpretation, $40 \%$ and $50 \%$ of the maximum tumor signal intensity, fixed SUV of 2.5, and the signal-to-background ratio method; Fig. 2; Table 1) (20). The results showed that the volume and shape of the resulting GTV were heavily influenced by the choice of the segmentation tool. Visual interpretation of the PET signal yielded volumes close to those of CT-based GTV delineation, whereas all automated segmentation methods resulted in significantly smaller GTVs than the GTVs based on clinical information and CT alone (20). Furthermore, in a large percentage of patients (between $29 \%$ and $64 \%$, depending on the segmentation tool used) more than $20 \%$ of the ${ }^{18} \mathrm{~F}$-FDG PET-based GTV was located outside the GTV based on clinical information and CT. This suggests that tumor could be identified by ${ }^{18}$ F-FDG PET that was missed using the standard methods of GTV delineation. However, in the absence of histologic validation it is unknown in what percentage of cases this was caused by
FIGURE 2. CT (left), ${ }^{18} \mathrm{~F}-\mathrm{FDG}$ PET (middle), and fused ${ }^{18} \mathrm{~F}-\mathrm{FDG}$ PET/CT (right) images of patient with T4N2M0 carcinoma of oral cavity (A) and patient with T4N2M0 carcinoma of base of tongue (B). Red = GTV delineated on CT (absolute volumes of $47.5 \mathrm{~cm}^{3}$ for A and $16.9 \mathrm{~cm}^{3}$ for B); green = PET-based GTVs obtained by visual interpretation (43.8 $\mathrm{cm}^{3}$ for $\mathrm{A}$ and $6.9 \mathrm{~cm}^{3}$ for $\mathrm{B}$ ); orange = GTVs obtained by applying isocontour of SUV of $2.5\left(32.6 \mathrm{~cm}^{3}\right.$ for A; this method was unsuccessful in $B$ because of inclusion of large areas with normal background signal); yellow = GTV obtained by using fixed threshold of $40 \%$ of maximum signal intensity $\left(20.1 \mathrm{~cm}^{3}\right.$ for A and $15.5 \mathrm{~cm}^{3}$ for B); dark blue = GTV obtained by using fixed threshold of $50 \%$ of maximum signal intensity $\left(14.9 \mathrm{~cm}^{3}\right.$ for A and $7.1 \mathrm{~cm}^{3}$ for B); light blue = GTV obtained by applying adaptive threshold based on signal-to-background ratio (15.7 $\mathrm{cm}^{3}$ for $A$ and $6.8 \mathrm{~cm}^{3}$ for $B$ ).

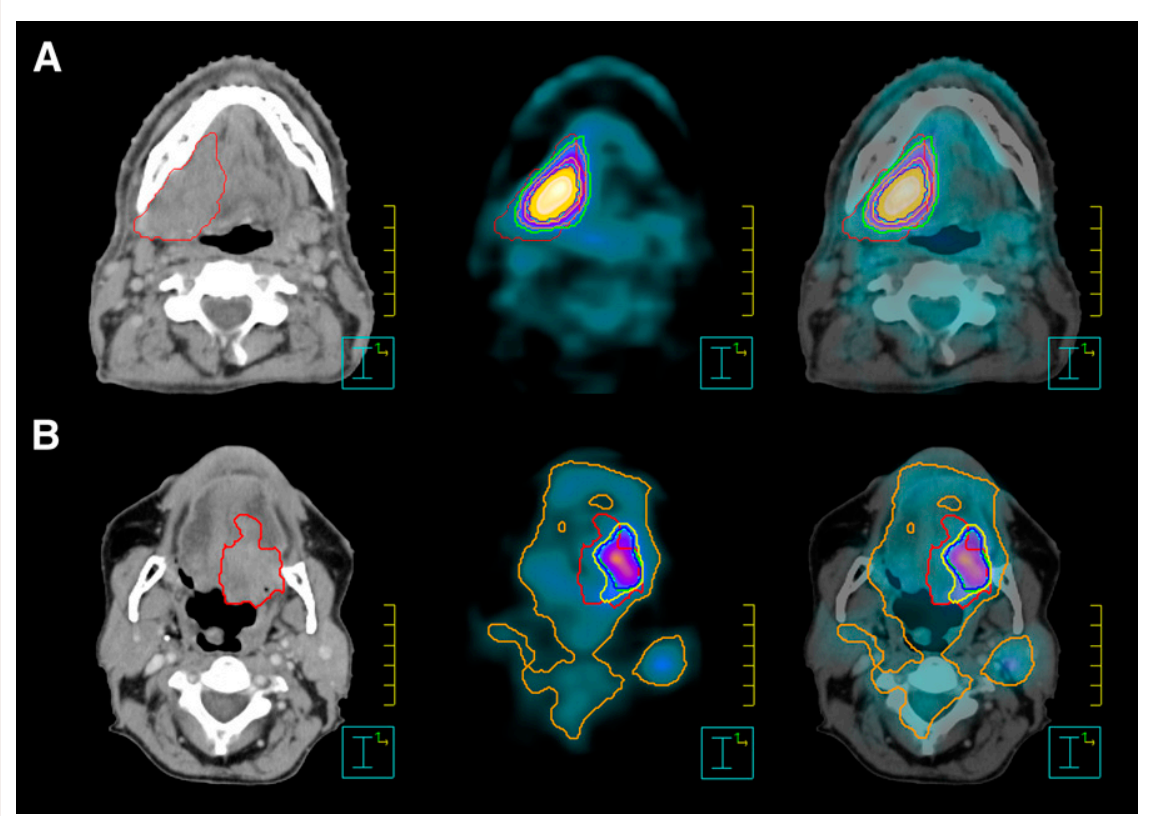


TABLE 1. Common Terminology Used in Radiation Therapy

Term

Gross tumor volume (GTV)

Clinical target volume

Planning target volume

Treatment volume

Metabolic tumor volume

Hypoxic tumor volume
Definition

Macroscopic tumor volume as detected by clinical examination and anatomic imaging (ultrasound, CT, MRI, PET)

GTV with margin added for subclinical microscopic spread

Clinical target volume with margin added for organ motion and setup inaccuracy

Tissue volume treated to substantial radiation dose (typically larger than planning target volume)

Metabolically active tumor volume as detected by biologic imaging, for example, ${ }^{18} \mathrm{~F}-\mathrm{FDG}$ PET

Hypoxic tumor volume as detected by biologic imaging, that is, hypoxia or hypoxia-related

PET tracers ( ${ }^{18} \mathrm{~F}-\mathrm{FMISO},{ }^{18} \mathrm{~F}$-fluoroazomycin arabinoside, ${ }^{18} \mathrm{~F}$-fluoroerythronitroimidazole), or blood oxygen level-dependent MRI peritumoral inflammation, resulting in a false-positive reading of the ${ }^{18} \mathrm{~F}$-FDG PET signal.

Most of the discussed data are based on theoretic delineation studies using operator-dependent or -independent segmentation tools. Only the study by Daisne et al. had results validated against histopathology (11). It is obvious that additional validation studies are needed as well as carefully designed clinical trials to address the issue of safety (side effects) and the clinical impact (locoregional control, survival) of incorporating PET for GTV delineation. However, it will be at least challenging to design and conduct such trials while PET/CT is increasingly incorporated into clinical practice on the basis of nonrandomized clinical studies in often relatively small patient populations.

Thus far, most delineation studies incorporating ${ }^{18} \mathrm{~F}$ FDG PET in head and neck cancer have concentrated on the primary tumor. This is probably due to the fact that CT-based delineation of metastatic lymph nodes is usually less prone to error because of better discrimination from the surrounding fatty tissue. However, this can be more difficult in cases with large, matted nodes. ${ }^{18} \mathrm{~F}-\mathrm{FDG}$ PET might be helpful in these situations, although one should be aware of the possibility of negative ${ }^{18} \mathrm{~F}-\mathrm{FDG}$ PET readings in necrotic parts of the lymph node.

Apart from more accurate target volume delineation for radiotherapy planning purposes, PET may identify parts of the GTV potentially requiring additional radiation doses. Assuming that ${ }^{18} \mathrm{~F}-\mathrm{FDG}$ uptake represents tumor cell density, ${ }^{18} \mathrm{~F}$-FDG PET can be used to direct dose escalation to ${ }^{18} \mathrm{~F}-\mathrm{FDG}-$ avid subvolumes of the tumor. The feasibility of this approach was demonstrated in various theoretic planning studies applying either uniform dose distribution or voxel-intensity-based IMRT $(21,22)$. The former method delivers a uniform escalated dose to an ${ }^{18} \mathrm{~F}-\mathrm{FDG}$-avid subvolume within the CT-based target volume. This approach was pioneered by Schwartz et al., who escalated total dose to $75 \mathrm{~Gy}$ in a theoretic planning study involving 20 patients with head and neck cancer (21). With voxelintensity-based IMRT, the ${ }^{18} \mathrm{~F}$-FDG signal intensity in the PET voxel is proportionally related to the dose prescribed to that voxel; that is, the higher the PET signal, the higher the prescribed dose (22). Both methods are alternatives for boosting ${ }^{18} \mathrm{~F}-\mathrm{FDG}$ PET subvolumes inside a CT-based planning target volume. The clinical feasibility of dose escalation using a uniform dose distribution was recently proven in a phase I clinical trial (23). Forty-one patients with head and neck cancer were treated with IMRT to dose levels of 72.5 and 77.5 Gy using a simultaneous integrated boost. With this technique, the escalated dose is delivered simultaneously with the lower dose to the low-risk areas, as opposed to being delivered sequentially, at the end of the treatment. Acute toxicity (dysphagia $\geq$ grade 3 ) occurred in $50 \%$ of patients at both dose levels, and dose-limiting toxicity was observed in 2 patients at the lower dose level and one patient at the higher. The authors concluded that PET-guided dose escalation appeared to be well tolerated, with high local control rates in both the lower-dose group and the higher-dose group ( $85 \%$ and $87 \%$, respectively) at 1 y of follow-up (Fig. 3) (23).

During the course of radiotherapy, the tumor volume gradually decreases and one might consider adjusting the GTV and ultimately the radiotherapy dose distribution accordingly. This adjustment could facilitate sparing of normal tissues. For example, during the treatment course for oropharyngeal tumors, the parotid gland is shifted centrally toward the high-dose region by tumor shrinkage and weight loss of the patient (24). As a result, a larger part of the parotid gland is potentially irradiated to a higher dose, possible resulting in a higher incidence and greater severity of xerostomia. Adaptive image-guided radiotherapy using repetitive PET/CT during the course of treatment is a promising approach to adjusting treatment volume and dose distribution, as has recently been demonstrated in a proofof-principle study (18). Throughout the course of radiotherapy, the GTVs based on ${ }^{18} \mathrm{~F}-\mathrm{FDG}$ PET significantly decreased and were at all times smaller than those defined using pretreatment CT and MRI. Radiation treatment planning based on ${ }^{18} \mathrm{~F}$-FDG PET and volume adaptation progressively reduced the irradiated volumes by $27 \%-42 \%$ $\left(\mathrm{V}_{90}-\mathrm{V}_{100}\right)$, compared with traditional CT-based treatment plans obtained before treatment. Disappointingly, this volume reduction only marginally affected the doses to the organs at risk, such as the parotid gland. Adaptive ${ }^{18} \mathrm{~F}$-FDG PET-guided radiotherapy nevertheless may be an attractive approach, especially for dose escalation strategies.

${ }^{18}$ F-FDG PET can provide important complementary information for radiotherapy planning in head and neck cancer. Potentially, the GTV can be reduced on the basis of 
FIGURE 3. Planning CT scan (A), corresponding ${ }^{18} \mathrm{~F}-\mathrm{FDG}$ PET fusion images ( $B$ and $C$ ), and calculated dose distribution (D) of patient with T3N2M0 hypopharyngeal carcinoma. Red = GTV delineated on CT (GTV CT $_{\text {; }}$ absolute volume of $39.0 \mathrm{~cm}^{3}$ ); light blue = GTV delineated on ${ }^{18} \mathrm{~F}$-FDG PET using adaptive threshold based on signal-to-background ratio (GTV $\mathrm{GBR}_{\mathrm{SBR}}$; absolute volume of $13.1 \mathrm{~cm}^{3}$ ). Also illustrated are planning target volume to 50.3 Gy (pink), to 68.0 Gy (dark blue), and to 72.0 Gy (light blue). Additional dose of 4.0 Gy is directed to GTV $_{\mathrm{SBR}}$ using IMRT with integrated boost technique in accelerated scheme. Subvolume of $\mathrm{GTV}_{\mathrm{CT}}$ thus receives total dose of 72.0 Gy.

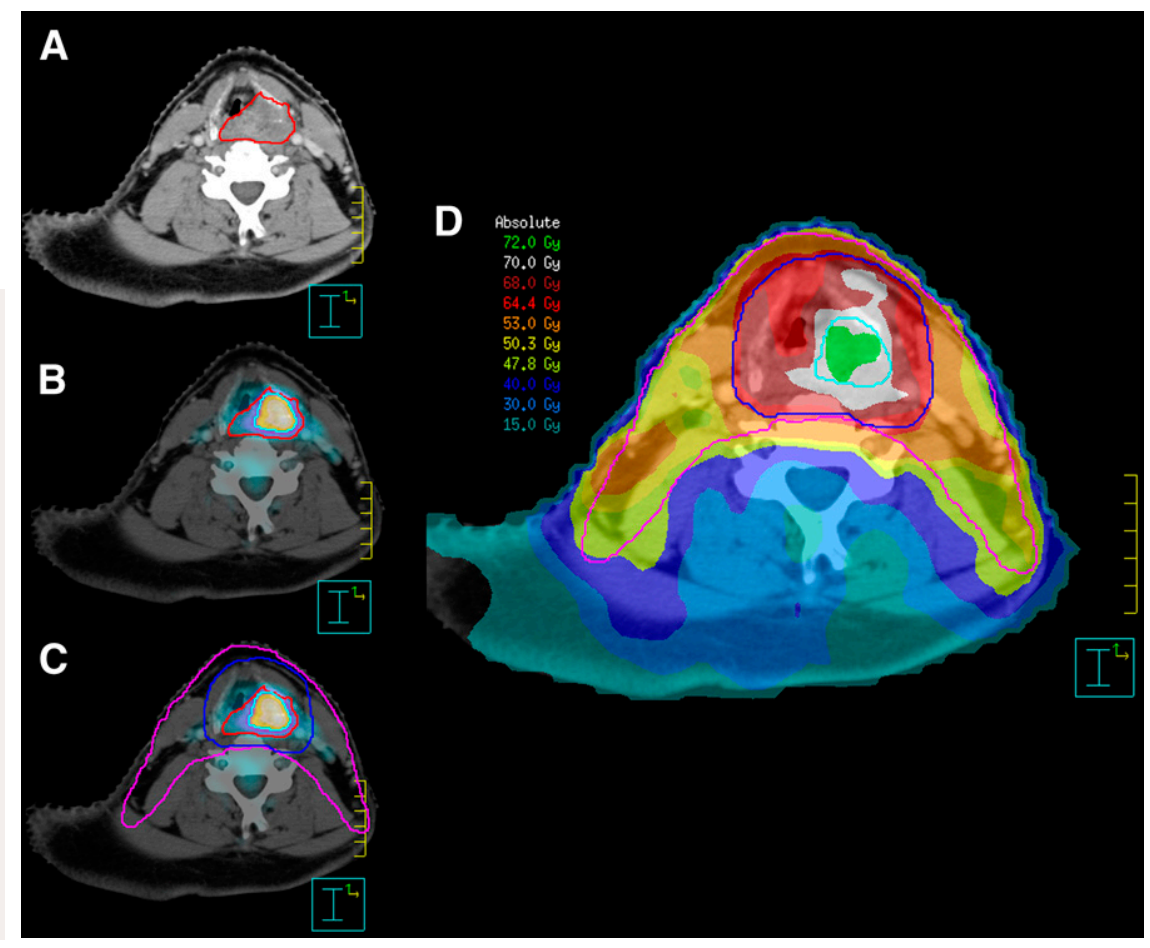

the PET information, facilitating sparing of nearby normal tissues and allowing dose escalation to relatively small subvolumes. Furthermore, biologic imaging using ${ }^{18} \mathrm{~F}-\mathrm{FDG}$ PET may identify areas of tumor spread not recognized by CT or MRI, potentially improving the accuracy of GTV definition. However, to address the clinical value and possible shortcomings of these concepts, additional histologic validation studies and properly designed clinical studies are needed.

Recently, the first results on clinical treatment outcome after integration of ${ }^{18} \mathrm{~F}-\mathrm{FDG} \mathrm{PET} / \mathrm{CT}$ data into IMRT planning have been published. In a case-control study, Rothschild et al. compared 45 patients with stage IV-A pharyngeal carcinomas treated with ${ }^{18} \mathrm{~F}-\mathrm{FDG}$ PET/CTbased IMRT with a matched historical cohort receiving standard 3-dimensional conformal radiotherapy (25). The 2-y overall survival and event-free survival rates of patients treated with ${ }^{18} \mathrm{~F}$-FDG PET/CT-based IMRT were $91 \%$ and $80 \%$ and significantly better than for the control group. In a similar study, Vernon et al. reported 2-y overall survival and disease-free survival rates of $83 \%$ and $71 \%$, respectively, for 42 patients with head and neck cancer of various stages and subsites (26). Toxicity profiles in this second study were reported as favorable.

Even though these initial results are encouraging, they must be interpreted cautiously because they are based on small and heterogeneous patient populations, they have only short follow-up periods, and they use historical controls. Furthermore, it remains unclear from both studies whether the suggested improvements in tumor control can be attributed to improved radiotherapy techniques or are due to the introduction of ${ }^{18} \mathrm{~F}$-FDG PET/CT or to other factors.

\section{IMAGING BIOLOGIC TUMOR CHARACTERISTICS RELEVANT TO RADIATION TREATMENT RESPONSE}

Three major tumor characteristics adversely affect treatment outcome and prognosis after radiation therapy: tumor cell hypoxia, repopulation during the course of treatment, and intrinsic radioresistance. These factors largely determine the outcome of radiotherapy in terms of local and regional tumor control but ultimately also the risk of distant metastases and survival. PET enables noninvasive biologic profiling of the tumor before and during radiation treatment, with the potential to tailor therapy according to individual characteristics.

\section{Hypoxia}

Hypoxia is a feature of many solid tumors and in particular squamous cell carcinomas of the cervix and the head and neck $(27,28)$. Tumor cell hypoxia can result from 2 mechanisms: limited diffusion capacity of oxygen due to a large distance from the supplying blood vessel (chronic hypoxia), or impaired perfusion of the supplying vessel due to temporary vasoconstriction or endovascular obstruction (acute hypoxia) (29). Treatment modifications are available, but at the cost of increased morbidity $(30,31)$. To individualize treatment and to select patients for these treatment modifications, assessment of the tumor oxygenation status is compulsory. In accessible tumors of the head and neck or uterine cervix, this assessment can be done by invasive polarographic electrode measurements or by immunohistochemical staining of markers in tumor biopsies (32-34). The advantage of the polarographic electrodes is that the entire tumor can be mapped using multiple tracks. However, its clinical use is limited by the invasive nature of the procedure, 
the restriction to accessible tumors, and the inability to distinguish between normal, necrotic, and tumor tissue. Immunohistochemical staining of tumor biopsy samples results in high-resolution images that can be analyzed for several endogenous and exogenous markers of interest. Unfortunately, the tumor biopsy samples are often small and represent only a fraction of the entire tumor. Furthermore, exogenous markers require intravenous administration before biopsy samples can be taken. Finally, the acquisition of a tumor biopsy often requires the use of general anesthesia, and this procedure is not attractive for repetitive measurement. Noninvasive imaging using PET can provide a spatial map of the intratumoral distribution of hypoxia before and during treatment. This information can potentially be used not only as a selection instrument for treatment modification but also for optimization of radiotherapy planning and delivery.

${ }^{18}$ F-fluoromisonidazole (FMISO) is a nitroimidazole PET tracer that is reduced and bound to cell constituents under hypoxic conditions. In the early 1990s, ${ }^{18}$ F-FMISO PET was applied in several small clinical trials on different primary tumors (35-37). Since then, ${ }^{18}$ F-FMISO PET has been extensively used for the detection of hypoxia in head and neck tumors (38-44). Importantly, in head and neck cancer it was shown that the level of hypoxia depicted by ${ }^{18}$ F-FMISO PET before treatment was correlated with locoregional failure $(38,42,45)$. Apart from its prognostic value, Rischin et al. published data supporting the predictive value of ${ }^{18} \mathrm{~F}$-FMISO PET (42). They performed ${ }^{18} \mathrm{~F}$ FMISO PET scans in patients with advanced-stage head and neck carcinomas that were treated with radiotherapy and concurrent chemotherapy alone or combined with a hypoxic cytotoxin. Patients with hypoxic primary tumors treated with the additional cytotoxin experienced significantly fewer local failures than patients treated with chemotherapy alone ( 0 of 8 patients vs. 6 of 9 ). Furthermore, the absence of hypoxia on ${ }^{18} \mathrm{~F}$-FMISO PET was associated with a low risk of locoregional failure when treated with chemotherapy alone (42). ${ }^{18}$ F-FMISO PET can thus serve as a predictive tool allowing treatment selection based on biologic tumor characteristics. Ultimately, reduction of side effects in patients not benefiting from treatment modification will be feasible.

Apart from tumor characterization, first attempts were made to delineate a biologic target volume and to escalate the dose to the primary tumor based on ${ }^{18} \mathrm{~F}$-FMISO PET (46-48). Two theoretic planning studies proved the feasibility of dose escalation to the ${ }^{18}$ F-FMISO PET-detected hypoxic subvolume using IMRT $(46,47)$. Rajendran et al. demonstrated that, using an IMRT technique, the dose to the ${ }^{18}$ F-FMISO PET-detected hypoxic subvolume could be escalated by an additional 10 Gy (47). Lee et al. achieved a dose of $84 \mathrm{~Gy}$ in hypoxic areas without exceeding the normal-tissue tolerance (46). Their attempt to further escalate the dose to $105 \mathrm{~Gy}$ in hypoxic regions was successful in only 1 of the 2 plans studied. In a third study, Thorwarth et al. compared IMRT planning with dose painting by numbers based on dynamic ${ }^{18} \mathrm{~F}$-FMISO PET data (48). Thereby, spatially variant doses are delivered to the tumor according to dose-escalation factors determined on the basis of the dynamic ${ }^{18}$ F-FMISO PET scan. With this approach, the tumor control probability was increased from $56 \%$ to $70 \%$ while the same level of toxicity was maintained (48). However, one has to be cautious in interpreting the data because the number of patients included in this study was small.

Until now, clinical experience with hypoxic PET tracers other than ${ }^{18} \mathrm{~F}$-FMISO has been limited. ${ }^{60} \mathrm{Cu}$ (II)-diacetylbis $\left(N^{4}\right.$-methylthiosemicarbazone $)$ was introduced into the clinic after successful preclinical studies demonstrating a strong correlation between tracer uptake and a low level for partial pressure of oxygen (49). It was the first hypoxiarelated PET tracer for which the potential use of a selective boost to the hypoxic subvolume was illustrated (50). However, partly because of its limited specificity, especially if imaging is performed at early time points after administration, this compound did not find its way into largerscale clinical studies.

${ }^{18} \mathrm{~F}$-fluoroerythronitroimidazole, ${ }^{18} \mathrm{~F}$-fluoroazomycin arabinoside, and ${ }^{18} \mathrm{~F}-2-(2-n i t r o i m i d a z o l-1-y l)-N-(3,3,3-$ trifluoropropyl)-acetamide $\left({ }^{18} \mathrm{~F}-\mathrm{EF} 3\right)$ are members of a new generation of nitroimidazoles. ${ }^{18} \mathrm{~F}$-fluoroerythronitroimidazole showed a higher and more heterogeneously distributed tracer uptake in tumors than in adjacent neck muscle (51). Furthermore, a high uptake of ${ }^{18}$ F-fluoroerythronitroimidazole before radiation therapy was associated with a trend toward poor overall survival (52). ${ }^{18} \mathrm{~F}$-fluoroazomycin arabinoside has similar tracer characteristics to ${ }^{18}$ F-fluoroerythronitroimidazole and was proven feasible and of sufficient quality for clinical use in patients with head and neck cancer $(53,54)$. Grosu et al. incorporated ${ }^{18} \mathrm{~F}$-fluoroazomycin arabinoside PET into radiation treatment planning and detected hypoxic subvolumes of different sizes and distributions (representing on average $11 \%$ of the primary tumor volume and $8 \%$ of the metastatic lymph node volume) (55). Dose escalation to $80.5 \mathrm{~Gy}$ in ${ }^{18} \mathrm{~F}$ fluoroazomycin arabinoside PET-detected hypoxic areas was shown to be feasible. ${ }^{18} \mathrm{~F}-\mathrm{EF} 3$ was used in a phase I study of patients with head and neck cancer (56). In that study, the use of this tracer was shown to be safe, but the number of advanced-stage tumors showing increased tracer uptake was disappointingly low.

In summary, although numerous hypoxic or hypoxiarelated PET tracers are available for clinical use, their prognostic and predictive value needs to be assessed in larger clinical studies before implementation for patient selection. Preferably, the PET tracer used must also visualize changes in the oxygenation status caused by treatment modifications counteracting hypoxia, such as carbogen breathing (57). More important, the concept of dose painting to hypoxic subvolumes either by uniform doses or by dose painting by numbers is still the subject of intense debate. There are major concerns about the spatial 
resolution of hypoxic PET when compared with the distribution and fluctuation of tumor cell hypoxia at the microregional level. In this context, we investigated 10 different head and neck carcinoma xenograft tumor lines using ${ }^{18}$ F-FMISO autoradiography and pimonidazole immunohistochemistry (Fig. 4) (58). We found that the pattern of the ${ }^{18} \mathrm{~F}$-FMISO signal depended on the distribution of hypoxia at the microregional level. In 5 xenograft tumor lines, a significant correlation between the mean ${ }^{18} \mathrm{~F}$ FMISO and pimonidazole signal intensities was found, and this correlation depended on the underlying microarchitecture. This finding indicates that one should be cautious when studying small tumor subvolumes for dose escalation (58). Apart from different distribution patterns of hypoxia at the microregional level, one has to consider that the oxygenation status changes during the course of

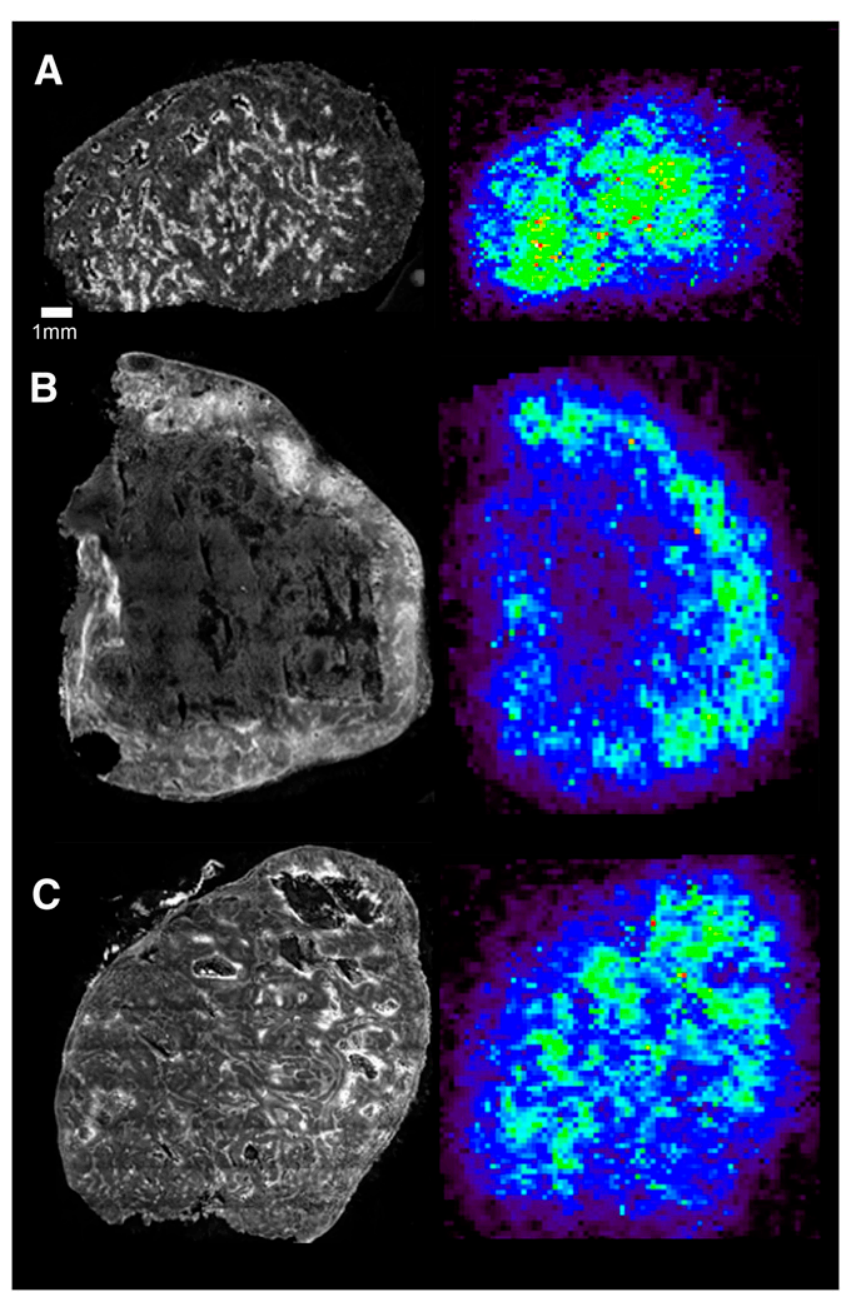

FIGURE 4. Gray-value images after immunohistochemical staining of nitroimidazole hypoxia marker pimonidazole (left) and ${ }^{18} \mathrm{~F}$-FMISO autoradiography images (right) of SCCNij3 (A), SCCNij153 (B), and SCCNij86 (C) xenografted human squamous cell carcinomas of head and neck. Corresponding staining patterns and signal intensities are seen for pimonidazole and ${ }^{18} \mathrm{~F}-\mathrm{FMISO}$ in SCCNij3 and SCNij153 but not in SCCNij86. radiotherapy, making repetitive PET imaging before and during treatment compulsory (59). Finally, the question on the radiation dose levels required for effective elimination of the radioresistant subpopulations remains unanswered.

\section{Tumor Cell Proliferation}

The major limitations of ${ }^{18} \mathrm{~F}$-FDG PET in oncology are false-positive readings due to tracer uptake in inflammatory tissue or reactive lymph nodes. Therefore, PET tracers that more specifically image DNA synthesis are being developed and tested.

Tumor cell proliferation during the course of therapy adversely affects radiation treatment outcome and prognosis in squamous cell carcinomas of the head and neck (60). $3^{\prime}$-deoxy-3 ${ }^{\prime}-{ }^{18} \mathrm{~F}$-fluorothymidine $\left({ }^{18} \mathrm{~F}\right.$-FLT) is a tracer that reflects the activity of thymidine kinase 1 , a principal enzyme in the salvage pathway of DNA synthesis (61). The ${ }^{18}$ F-FLT PET signal is more specific for actively dividing tumor cells than is the ${ }^{18}$ F-FDG PET signal. Inflammatory cells near the tumor consume glucose and thus cause false-positive ${ }^{18}$ F-FDG PET readings. However, as these immune response cells are terminally differentiated, the DNA synthesis rate and therefore the ${ }^{18} \mathrm{~F}$-FLT uptake are not increased. ${ }^{18}$ F-FLT PET was validated against histopathology in a variety of solid tumors including breast, lung, and sarcoma (62-64). In soft-tissue sarcoma, Cobben et al. found a significant correlation between the SUVs and labeling index of the proliferation marker Ki-67. In addition, ${ }^{18}$ F-FLT PET was able to distinguish low-grade from high-grade soft-tissue sarcomas (62). In breast tumors, Kenny et al. reported a strong correlation between SUVs and the fully quantitative net irreversible plasma to tumor transfer constant $\left(\mathrm{K}_{\mathrm{i}}\right)$ parameter of dynamic ${ }^{18}$ F-FLT PET and staining of Ki-67 (63). Finally, Yap et al. also observed a significant correlation between ${ }^{18}$ F-FLT uptake in non-small cell lung cancer lesions and the Ki-67 labeling index (64). In primary head and neck tumors, this promising compound has thus far been applied only to primary laryngeal tumors (65). Validation of ${ }^{18}$ F-FLT PET in a large series of squamous cell carcinomas of the head and neck is ongoing at our center. For the detection of cervical lymph node metastases, first results demonstrated that ${ }^{18} \mathrm{~F}$-FLT PET is not suitable in this tumor entity (66). A high rate of false-positive findings caused by ${ }^{18}$ F-FLT uptake in the germinal centers of reactive lymph nodes resulted in a low specificity and a low positive predictive value (17\% and 38\%, respectively) (66).

Until the present, adaptive image-guided radiotherapy has been based on repetitive PET scanning using ${ }^{18} \mathrm{~F}-\mathrm{FDG}$ (18). As the treatment course progresses, the obtained ${ }^{18} \mathrm{~F}-$ FDG PET signal is heavily influenced by the inflammatory response of tumor-surrounding tissues, leading to an increased background activity. As a result, segmentation of the PET signal for tumor delineation purposes becomes increasingly difficult. The use of a proliferation-specific PET tracer, such as ${ }^{18} \mathrm{~F}-\mathrm{FLT}$, may be a solution to this 
problem. During the course of therapy, the reduction in the proliferative activity of the primary tumor can be accurately imaged by ${ }^{18} \mathrm{~F}$-FLT, not disturbed by increased tracer uptake in surrounding inflammatory tissue. Our group is assessing the changes in the ${ }^{18} \mathrm{~F}$-FLT PET signal during therapy in patients with squamous cell carcinomas of the head and neck treated with radiotherapy alone or with concomitant chemotherapy (Fig. 5). The predictive potential of this approach and applicability for tailored treatment are the subject of investigation.

\section{Perfusion, Protein Synthesis, and Others}

Another significant tumor characteristic strongly related to tumor cell hypoxia is tumor blood perfusion. Hypoxia is a strong stimulus for neovascularization, but many newly formed vessels are of poor quality and have severe structural and functional abnormalities. Despite increased vascular density, the impaired functionality of blood vessels may result in deprivation of oxygen and nutrients. Therefore, an imaging tool for assessment of tumor blood flow may provide important information relevant for radiotherapy responsiveness. Lehtiö et al. used ${ }^{15} \mathrm{O}$-labeled water $\left({ }^{15} \mathrm{O}-\mathrm{H}_{2} \mathrm{O}\right)$ and ${ }^{18} \mathrm{~F}$-fluoroerythronitroimidazole for imaging of perfusion and hypoxia in 21 patients with head and neck cancer (51). Preliminary results from this small study indicated an association between tumor perfusion and radiation treatment outcome (52).

$O-2-{ }^{18} \mathrm{~F}$-fluoroethyl-L-tyrosine $\left({ }^{18} \mathrm{~F}\right.$-FET $)$ and L-methyl${ }^{11} \mathrm{C}$-methionine $\left({ }^{11} \mathrm{C}\right.$-MET) are amino acid analogs used to visualize cellular amino acid uptake or protein synthesis. ${ }^{18} \mathrm{~F}$-FET may be useful in differentiating tumor from posttreatment inflammatory tissue, as it is not taken up by inflammatory cells. Several studies compared ${ }^{18}$ F-FET with ${ }^{18} \mathrm{~F}-\mathrm{FDG}$ PET in squamous cell carcinomas of the head and neck and histopathologically confirmed the specific uptake of ${ }^{18}$ F-FET by malignant cells (67-69). The specificity of
${ }^{18} \mathrm{~F}$-FET PET was found to be superior to that of ${ }^{18} \mathrm{~F}-\mathrm{FDG}$ PET $(95 \%-100 \%$ vs. $63 \%-79 \%)$, but the sensitivity of the amino acid tracer was significantly lower $(64 \%-75 \%$ vs. $93 \%-95 \%$, respectively). Because the SUVs for ${ }^{18} \mathrm{~F}$-FET PET were significantly lower than those for ${ }^{18} \mathrm{~F}$-FDG PET, the new tracer will probably not replace ${ }^{18} \mathrm{~F}$-FDG PET as a diagnostic tool but can provide complementary information for discrimination between tumor and inflammatory tissue (67-69).

${ }^{11} \mathrm{C}$-MET PET is similar in sensitivity and specificity to ${ }^{18}$ F-FDG PET (70). In a delineation study, ${ }^{11} \mathrm{C}$-MET was compared with ${ }^{18} \mathrm{~F}$-FDG PET and CT. Although, compared with CT, ${ }^{18}$ F-FDG PET yielded significantly smaller GTVs, GTVs based on ${ }^{11} \mathrm{C}$-MET PET were not different from GTVs based on CT, probably because of uptake by surrounding normal mucosa and salivary gland tissue. The authors concluded that ${ }^{11} \mathrm{C}$-MET has no additional value for target volume delineation in head and neck tumors (71).

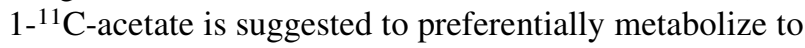
the membrane lipids in tumor cells. In a staging and radiotherapy planning study for head and neck cancer, $1-{ }^{11} \mathrm{C}$-acetate PET detected all primary tumors and $95 \%$ of the metastatic lymph nodes, more than ${ }^{18} \mathrm{~F}$-FDG PET and CT/MRI (72). However, the GTVs derived by $1-{ }^{11} \mathrm{C}$-acetate PET were $51 \%$ larger than those based on ${ }^{18} \mathrm{~F}$-FDG PET. Before $1-{ }^{11} \mathrm{C}$-acetate PET can be introduced in the radiotherapy planning process, further studies are needed to explain this discrepancy and to clarify the mechanism of tumor uptake.

Finally, noninvasive methods to assess the uptake and biodistribution of biologic modifiers will be of great value to direct new targeted therapies. Radiolabeled antibodies and small molecules for PET are currently being developed and tested in preclinical and early clinical studies $(73,74)$.

In conclusion, PET tracers that image specific biologic tumor characteristics offer potential for tailor-made radia-

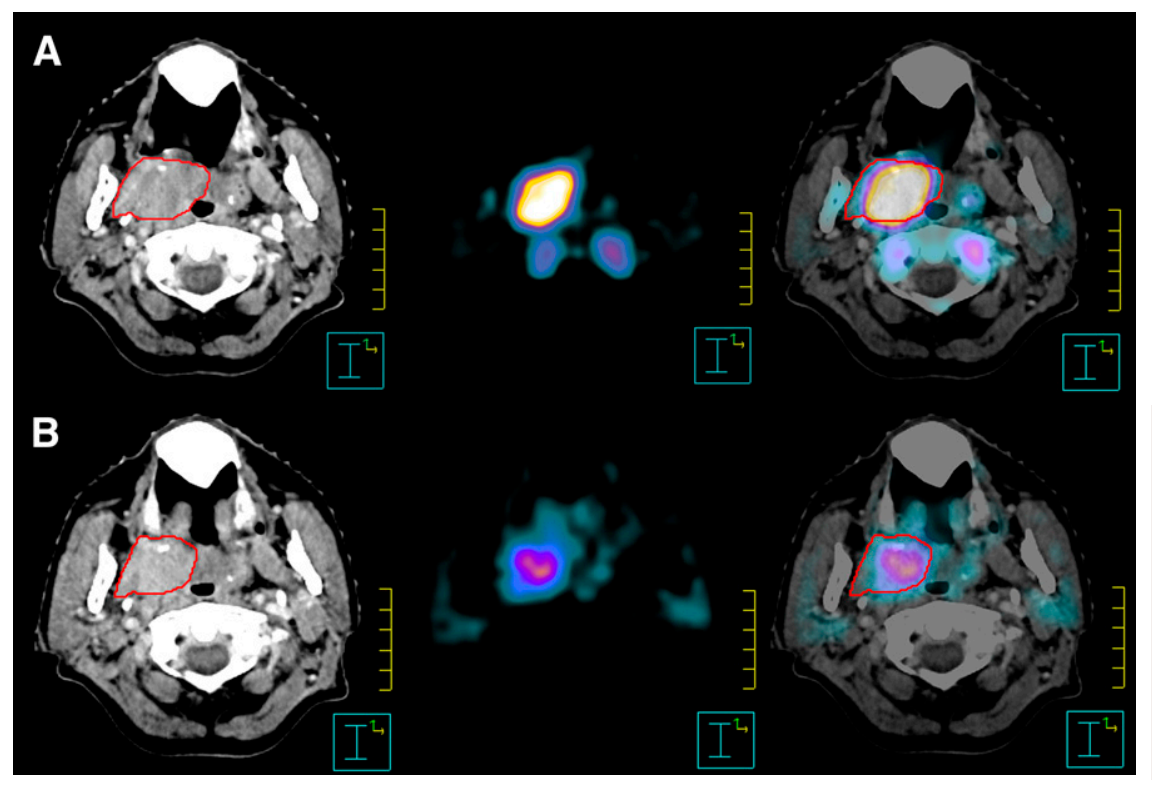

FIGURE 5. CT (left), ${ }^{18} \mathrm{~F}-\mathrm{FLT}$ PET (middle), and fused ${ }^{18} \mathrm{~F}-\mathrm{FLT}$ PET/CT (right) images of patient with T3NOMO oropharyngeal carcinoma before radiation therapy $(A)$ and after 8 fractions of 2 Gy (B). Red = GTV based on CT. In B, significant reduction in ${ }^{18} \mathrm{~F}-\mathrm{FLT}$ PET signal intensity is already seen, whereas only a modest decrease of GTV based on CT is seen at this dose level. 
tion therapy. However, they remain in the research arena until proper clinical validation has occurred.

\section{TECHNICAL INNOVATIONS}

Several challenges regarding PET scanning remain, of which some may be resolved or improved whereas others cannot. For example, resolution is limited by the distance a positron travels before it annihilates. This distance is a given fact for a certain radionuclide positron emitter and therefore unchangeable. Furthermore, various developments regarding an increase in spatial and temporal resolution are ongoing. Currently, the spatial resolution for human PET scanners is on the order of 5-7 mm, compared with 1-3 $\mathrm{mm}$ for small-animal scanners. New developments in the size of the detector crystal, the coincidence timing window, and signal processing have achieved a resolution of $2 \mathrm{~mm}$ for human applications (75). These developments reduce image distortion and blurring and may increase the precision of tumor delineation.

Integrated PET/MRI scanners combine anatomic with functional imaging and may have a specific impact on the staging and treatment of head and neck cancer (76). The potential benefits of integrated PET/CT for the planning of radiotherapy have been discussed in this review. However, for particular subsites of the head and neck region, such as oropharyngeal and oral cavity tumors, MRI is the diagnostic imaging modality of choice. In these tumor sites, integrated PET/MRI scanners may further improve the accuracy of GTV delineation. In addition, dynamic MRI studies such as dynamic contrast-enhanced MRI and blood oxygen level-dependent MRI, as well as MR spectroscopy, may add complementary functional information.

\section{CONCLUSION}

${ }^{18}$ F-FDG PET is the gold standard for noninvasive functional imaging in oncology. In head and neck tumors, ${ }^{18} \mathrm{~F}$-FDG PET is not recommended for detection of the primary tumor, and its value for metastatic lymph nodes is still a matter of debate. With regard to staging of the primary tumor, ${ }^{18} \mathrm{~F}$-FDG PET may influence the treatment decision if distant metastases or second primary tumors are detected.

For radiotherapy planning in head and neck cancer, ${ }^{18} \mathrm{~F}$ FDG PET can provide important information complementary to CT. On the basis of PET information, the volume irradiated to high dose-levels may be reduced, thus facilitating the sparing of normal structures and the escalation of dose. However, additional histologic validation studies and properly designed clinical studies are needed to address the clinical value and possible shortcomings of this concept. Several PET tracers that image biologic tumor characteristics reflecting radiation resistance mechanisms are available and offer potential for tailored radiation therapy. However, they should be restricted to research purposes until proper clinical validation has occurred. In this context, the use of more than one tracer may open new horizons in the future. Finally, technical developments in PET scanning in general and in the field of head and neck cancer in particular may increase the precision of radiotherapy planning and thus improve tumor control and reduce treatment-related morbidity.

\section{ACKNOWLEDGMENTS}

This work was supported by EC FP6 funding (Biocare contract LSHC-CT-2004-505785) and by Junior Investigator Grant 2006-38 of the Radboud University Nijmegen Medical Centre, the Netherlands. We thank Peter van Kollenburg and Aswin Hoffmann for their contribution to figure design.

\section{REFERENCES}

1. Hunt MA, Zelefsky MJ, Wolden S, et al. Treatment planning and delivery of intensity-modulated radiation therapy for primary nasopharynx cancer. Int $J$ Radiat Oncol Biol Phys. 2001;49:623-632.

2. Kam MK, Chau RM, Suen J, Choi PH, Teo PM. Intensity-modulated radiotherapy in nasopharyngeal carcinoma: dosimetric advantage over conventional plans and feasibility of dose escalation. Int J Radiat Oncol Biol Phys. 2003;56:145-157.

3. Fletcher JW, Djulbegovic B, Soares HP, et al. Recommendations on the use of ${ }^{18}$ F-FDG PET in oncology. J Nucl Med. 2008;49:480-508.

4. Kyzas PA, Evangelou E, Denaxa-Kyza D, Ioannidis JP. ${ }^{18}$ F-fluorodeoxyglucose positron emission tomography to evaluate cervical node metastases in patients with head and neck squamous cell carcinoma: a meta-analysis. J Natl Cancer Inst. 2008;100:712-720.

5. Abgral R, Querellou S, Potard G, et al. Does ${ }^{18}$ F-FDG PET/CT improve the detection of posttreatment recurrence of head and neck squamous cell carcinoma in patients negative for disease on clinical follow-up? J Nucl Med. 2009;50: 24-29.

6. Vermeersch H, Loose D, Ham H, Otte A, Van de Wiele C. Nuclear medicine imaging for the assessment of primary and recurrent head and neck carcinoma using routinely available tracers. Eur J Nucl Med Mol Imaging. 2003;30:1689-1700.

7. Schinagl DA, Kaanders JH, Oyen WJ. From anatomical to biological target volumes: the role of PET in radiation treatment planning. Cancer Imaging. 2006;6(suppl):S107-S116.

8. Steenbakkers RJ, Duppen JC, Fitton I, et al. Reduction of observer variation using matched CT-PET for lung cancer delineation: a three-dimensional analysis. Int J Radiat Oncol Biol Phys. 2006;64:435-448.

9. Ciernik IF, Dizendorf E, Baumert BG, et al. Radiation treatment planning with an integrated positron emission and computer tomography (PET/CT): a feasibility study. Int J Radiat Oncol Biol Phys. 2003;57:853-863.

10. Riegel AC, Berson AM, Destian S, et al. Variability of gross tumor volume delineation in head-and-neck cancer using CT and PET/CT fusion. Int J Radiat Oncol Biol Phys. 2006;65:726-732.

11. Daisne JF, Duprez T, Weynand B, et al. Tumor volume in pharyngolaryngeal squamous cell carcinoma: comparison at CT, MR imaging, and FDG PET and validation with surgical specimen. Radiology. 2004;233:93-100.

12. Heron DE, Andrade RS, Flickinger J, et al. Hybrid PET-CT simulation for radiation treatment planning in head-and-neck cancers: a brief technical report. Int J Radiat Oncol Biol Phys. 2004;60:1419-1424.

13. Nishioka T, Shiga T, Shirato H, et al. Image fusion between ${ }^{18}$ FDG-PET and MRI/CT for radiotherapy planning of oropharyngeal and nasopharyngeal carcinomas. Int J Radiat Oncol Biol Phys. 2002;53:1051-1057.

14. Nestle U, Kremp S, Schaefer-Schuler A, et al. Comparison of different methods for delineation of ${ }^{18} \mathrm{~F}$-FDG PET-positive tissue for target volume definition in radiotherapy of patients with non-small cell lung cancer. J Nucl Med. 2005;46:1342-1348.

15. Paulino AC, Koshy M, Howell R, Schuster D, Davis LW. Comparison of CT- and FDG-PET-defined gross tumor volume in intensity-modulated radiotherapy for head-and-neck cancer. Int J Radiat Oncol Biol Phys. 2005;61:1385-1392.

16. Daisne JF, Sibomana M, Bol A, Doumont T, Lonneux M, Gregoire V. Tridimensional automatic segmentation of PET volumes based on measured source- 
to-background ratios: influence of reconstruction algorithms. Radiother Oncol. 2003;69:247-250.

17. Geets X, Lee JA, Bol A, Lonneux M, Gregoire V. A gradient-based method for segmenting FDG-PET images: methodology and validation. Eur J Nucl Med Mol Imaging. 2007;34:1427-1438.

18. Geets X, Tomsej M, Lee JA, et al. Adaptive biological image-guided IMRT with anatomic and functional imaging in pharyngo-laryngeal tumors: impact on target volume delineation and dose distribution using helical tomotherapy. Radiother Oncol. 2007;85:105-115.

19. van Dalen JA, Hoffmann AL, Dicken V, et al. A novel iterative method for lesion delineation and volumetric quantification with FDG PET. Nucl Med Commun. 2007;28:485-493.

20. Schinagl DA, Vogel WV, Hoffmann AL, van Dalen JA, Oyen WJ, Kaanders JH. Comparison of five segmentation tools for ${ }^{18} \mathrm{~F}$-fluoro-deoxy-glucose-positron emission tomography-based target volume definition in head and neck cancer. Int J Radiat Oncol Biol Phys. 2007;69:1282-1289.

21. Schwartz DL, Ford EC, Rajendran J, et al. FDG-PET/CT-guided intensity modulated head and neck radiotherapy: a pilot investigation. Head Neck. 2005;27:478-487.

22. Vanderstraeten B, Duthoy W, De Gersem W, De Neve W, Thierens H. $\left[{ }^{18} \mathrm{~F}\right]$ fluoro-deoxy-glucose positron emission tomography $\left(\left[{ }^{18} \mathrm{~F}\right] \mathrm{FDG}-\mathrm{PET}\right)$ voxel intensity-based intensity-modulated radiation therapy (IMRT) for head and neck cancer. Radiother Oncol. 2006;79:249-258.

23. Madani I, Duthoy W, Derie C, et al. Positron emission tomography-guided, focal-dose escalation using intensity-modulated radiotherapy for head and neck cancer. Int J Radiat Oncol Biol Phys. 2007;68:126-135.

24. Barker JL Jr, Garden AS, Ang KK, et al. Quantification of volumetric and geometric changes occurring during fractionated radiotherapy for head-and-neck cancer using an integrated CT/linear accelerator system. Int J Radiat Oncol Biol Phys. 2004;59:960-970.

25. Rothschild S, Studer G, Seifert B, et al. PET/CT staging followed by intensitymodulated radiotherapy (IMRT) improves treatment outcome of locally advanced pharyngeal carcinoma: a matched-pair comparison. Radiat Oncol. 2007;2:22.

26. Vernon MR, Maheshwari M, Schultz CJ, et al. Clinical outcomes of patients receiving integrated PET/CT-guided radiotherapy for head and neck carcinoma. Int J Radiat Oncol Biol Phys. 2008;70:678-684.

27. Brizel DM, Sibley GS, Prosnitz LR, Scher RL, Dewhirst MW. Tumor hypoxia adversely affects the prognosis of carcinoma of the head and neck. Int J Radiat Oncol Biol Phys. 1997;38:285-289.

28. Hockel M, Schlenger K, Mitze M, Schaffer U, Vaupel P. Hypoxia and radiation response in human tumors. Semin Radiat Oncol. 1996;6:3-9.

29. Thomlinson RH, Gray LH. The histological structure of some human lung cancers and the possible implications for radiotherapy. Br J Cancer. 1955;9: 539-549.

30. Kaanders JH, Pop LA, Marres HA, et al. ARCON: experience in 215 patients with advanced head-and-neck cancer. Int $J$ Radiat Oncol Biol Phys. 2002;52:769-778.

31. Overgaard J, Hansen HS, Overgaard M, et al. A randomized double-blind phase III study of nimorazole as a hypoxic radiosensitizer of primary radiotherapy in supraglottic larynx and pharynx carcinoma: results of the Danish Head and Neck Cancer Study (DAHANCA) protocol 5-85. Radiother Oncol. 1998;46: 135-146.

32. Bussink J, Kaanders JH, van der Kogel AJ. Tumor hypoxia at the micro-regional level: clinical relevance and predictive value of exogenous and endogenous hypoxic cell markers. Radiother Oncol. 2003;67:3-15.

33. Kaanders JH, Wijffels KI, Marres HA, et al. Pimonidazole binding and tumor vascularity predict for treatment outcome in head and neck cancer. Cancer Res. 2002;62:7066-7074.

34. Nordsmark M, Loncaster J, Chou SC, et al. Invasive oxygen measurements and pimonidazole labeling in human cervix carcinoma. Int J Radiat Oncol Biol Phys. 2001;49:581-586.

35. Koh WJ, Rasey JS, Evans ML, et al. Imaging of hypoxia in human tumors with [F-18]fluoromisonidazole. Int J Radiat Oncol Biol Phys. 1992;22:199-212.

36. Rasey JS, Koh WJ, Evans ML, et al. Quantifying regional hypoxia in human tumors with positron emission tomography of $\left[{ }^{18} \mathrm{~F}\right]$ fluoromisonidazole: a pretherapy study of 37 patients. Int J Radiat Oncol Biol Phys. 1996;36:417-428.

37. Valk PE, Mathis CA, Prados MD, Gilbert JC, Budinger TF. Hypoxia in human gliomas: demonstration by PET with fluorine-18-fluoromisonidazole. $\mathrm{J} \mathrm{Nucl}$ Med. 1992;33:2133-2137.

38. Eschmann SM, Paulsen F, Reimold M, et al. Prognostic impact of hypoxia imaging with ${ }^{18} \mathrm{~F}$-misonidazole PET in non-small cell lung cancer and head and neck cancer before radiotherapy. J Nucl Med. 2005;46:253-260.

39. Gagel B, Reinartz P, Dimartino E, et al. $\mathrm{pO}_{2}$ ) polarography versus positron emission tomography $\left(\left[{ }^{18} \mathrm{~F}\right]\right.$ fluoromisonidazole, $\left[{ }^{18} \mathrm{~F}\right]-2$-fluoro- $2^{\prime}$-deoxyglucose): an appraisal of radiotherapeutically relevant hypoxia. Strahlenther Onkol. 2004; 180:616-622.

40. Hicks RJ, Rischin D, Fisher R, Binns D, Scott AM, Peters LJ. Utility of FMISO PET in advanced head and neck cancer treated with chemoradiation incorporating a hypoxia-targeting chemotherapy agent. Eur J Nucl Med Mol Imaging. 2005;32:1384-1391.

41. Rajendran JG, Mankoff DA, O'Sullivan F, et al. Hypoxia and glucose metabolism in malignant tumors: evaluation by $\left[{ }^{18} \mathrm{~F}\right]$ fluoromisonidazole and $\left[{ }^{18} \mathrm{~F}\right]$ fluorodeoxyglucose positron emission tomography imaging. Clin Cancer Res. 2004;10:2245-2252.

42. Rischin D, Hicks RJ, Fisher R, et al. Prognostic significance of $\left[{ }^{18} \mathrm{~F}\right]-$ misonidazole positron emission tomography-detected tumor hypoxia in patients with advanced head and neck cancer randomly assigned to chemoradiation with or without tirapazamine: a substudy of Trans-Tasman Radiation Oncology Group Study 98.02. J Clin Oncol. 2006;24:2098-2104.

43. Thorwarth D, Eschmann SM, Paulsen F, Alber M. A kinetic model for dynamic $\left[{ }^{18} \mathrm{~F}\right]$-Fmiso PET data to analyse tumour hypoxia. Phys Med Biol. 2005;50:22092224.

44. Thorwarth D, Eschmann SM, Holzner F, Paulsen F, Alber M. Combined uptake of $\left[{ }^{18} \mathrm{~F}\right] \mathrm{FDG}$ and $\left[{ }^{18} \mathrm{~F}\right] \mathrm{FMISO}$ correlates with radiation therapy outcome in headand-neck cancer patients. Radiother Oncol. 2006;80:151-156.

45. Thorwarth D, Eschmann SM, Scheiderbauer J, Paulsen F, Alber M. Kinetic analysis of dynamic ${ }^{18} \mathrm{~F}$-fluoromisonidazole PET correlates with radiation treatment outcome in head-and-neck cancer. BMC Cancer. 2005;5:152.

46. Lee NY, Mechalakos JG, Nehmeh S, et al. Fluorine-18-labeled fluoromisonidazole positron emission and computed tomography-guided intensity-modulated radiotherapy for head and neck cancer: a feasibility study. Int $J$ Radiat Oncol Biol Phys. 2008;70:2-13.

47. Rajendran JG, Hendrickson KR, Spence AM, Muzi M, Krohn KA, Mankoff DA. Hypoxia imaging-directed radiation treatment planning. Eur J Nucl Med Mol Imaging. 2006;33(suppl 1):44-53.

48. Thorwarth D, Eschmann SM, Paulsen F, Alber M. Hypoxia dose painting by numbers: a planning study. Int J Radiat Oncol Biol Phys. 2007;68:291-300.

49. Lewis JS, McCarthy DW, McCarthy TJ, Fujibayashi Y, Welch MJ. Evaluation of ${ }^{64} \mathrm{Cu}$-ATSM in vitro and in vivo in a hypoxic tumor model. J Nucl Med. 1999;40:177183.

50. Chao KS, Bosch WR, Mutic S, et al. A novel approach to overcome hypoxic tumor resistance: Cu-ATSM-guided intensity-modulated radiation therapy. Int J Radiat Oncol Biol Phys. 2001;49:1171-1182.

51. Lehtiö K, Oikonen V, Gronroos T, et al. Imaging of blood flow and hypoxia in head and neck cancer: initial evaluation with $\left[{ }^{15} \mathrm{O}^{\mathrm{O}} \mathrm{H}_{2} \mathrm{O}\right.$ and $\left[{ }^{18} \mathrm{~F}\right]$ fluoroerythronitroimidazole PET. J Nucl Med. 2001;42:1643-1652.

52. Lehtio K, Eskola O, Viljanen T, et al. Imaging perfusion and hypoxia with PET to predict radiotherapy response in head-and-neck cancer. Int J Radiat Oncol Biol Phys. 2004;59:971-982.

53. Kumar P, Stypinski D, Xia H, McEwan AJB, Machulla HJ, Wiebe LI. Fluoroazomycine arabinoside (FAZA): synthesis, ${ }^{2} \mathrm{H}$ and ${ }^{3} \mathrm{H}$-labelling and preliminary biological evaluation of a novel 2-nitroimidazole marker of tissue hypoxia. J Labelled Comp Radiopharm. 1999;42:3-16.

54. Souvatzoglou M, Grosu AL, Roper B, et al. Tumour hypoxia imaging with $\left[{ }^{18}\right.$ F]FAZA PET in head and neck cancer patients: a pilot study. Eur J Nucl Med Mol Imaging. 2007;34:1566-1575.

55. Grosu AL, Souvatzoglou M, Roper B, et al. Hypoxia imaging with FAZA-PET and theoretical considerations with regard to dose painting for individualization of radiotherapy in patients with head and neck cancer. Int J Radiat Oncol Biol Phys. 2007;69:541-551.

56. Mahy P, Geets X, Lonneux M, et al. Determination of tumour hypoxia with $\left[\left({ }^{18}\right) \mathrm{F}\right] \mathrm{EF} 3$ in patients with head and neck tumours: a phase I study to assess the tracer pharmacokinetics, biodistribution and metabolism. Eur J Nucl Med Mol Imaging. 2008;35:1282-1289.

57. Troost EG, Laverman $\mathrm{P}$, Kaanders JH, et al. Imaging hypoxia after oxygenationmodification: comparing $\left[{ }^{18} \mathrm{~F}\right] \mathrm{FMISO}$ autoradiography with pimonidazole immunohistochemistry in human xenograft tumors. Radiother Oncol. 2006;80: 157-164.

58. Troost EG, Laverman P, Philippens ME, et al. Correlation of $\left[\left({ }^{18}\right)\right.$ F $]$ FMISO autoradiography and pimonidazole immunohistochemistry in human head and neck carcinoma xenografts. Eur J Nucl Med Mol Imaging. 2008;35:1803-1811.

59. Nehmeh SA, Lee NY, Schroder H, et al. Reproducibility of intratumor distribution of ${ }^{18} \mathrm{~F}$-fluoromisonidazole in head and neck cancer. Int $J$ Radiat Oncol Biol Phys. 2008;70:235-242.

60. Kim JJ, Tannock IF. Repopulation of cancer cells during therapy: an important cause of treatment failure. Nat Rev Cancer. 2005;5:516-525.

61. Shields AF, Grierson JR, Dohmen BM, et al. Imaging proliferation in vivo with [F-18]FLT and positron emission tomography. Nat Med. 1998;4:1334-1336. 
62. Cobben DC, Elsinga PH, Suurmeijer AJ, et al. Detection and grading of soft tissue sarcomas of the extremities with ${ }^{18} \mathrm{~F}-3^{\prime}$-fluoro-3'-deoxy-L-thymidine. Clin Cancer Res. 2004;10:1685-1690.

63. Kenny LM, Vigushin DM, Al-Nahhas A, et al. Quantification of cellular proliferation in tumor and normal tissues of patients with breast cancer by $\left[{ }^{18} \mathrm{~F}\right]$ fluorothymidine-positron emission tomography imaging: evaluation of analytical methods. Cancer Res. 2005;65:10104-10112.

64. Yap CS, Czernin J, Fishbein MC, et al. Evaluation of thoracic tumors with ${ }^{18} \mathrm{~F}$ fluorothymidine and ${ }^{18} \mathrm{~F}$-fluorodeoxyglucose-positron emission tomography. Chest. 2006;129:393-401.

65. Cobben DC, van der Laan BF, Maas B, et al. ${ }^{18}$ F-FLT PET for visualization of laryngeal cancer: comparison with ${ }^{18}$ F-FDG PET. J Nucl Med. 2004;45:226-231.

66. Troost EG, Vogel WV, Merkx MA, et al. ${ }^{18}$ F-FLT PET does not discriminate between reactive and metastatic lymph nodes in primary head and neck cancer patients. J Nucl Med. 2007;48:726-735.

67. Balogova S, Perie S, Kerrou K, et al. Prospective comparison of FDG and FET $\mathrm{PET} / \mathrm{CT}$ in patients with head and neck squamous cell carcinoma. Mol Imaging Biol. 2008;10:364-373.

68. Pauleit D, Stoffels G, Schaden W, et al. PET with O-(2- ${ }^{18}$ F-fluoroethyl)-L-tyrosine in peripheral tumors: first clinical results. J Nucl Med. 2005;46:411-416.

69. Pauleit D, Zimmermann A, Stoffels G, et al. ${ }^{18}$ F-FET PET compared with ${ }^{18}$ F-FDG PET and CT in patients with head and neck cancer. $J$ Nucl Med. 2006;47:256-261.
70. Leskinen-Kallio S, Lindholm P, Lapela M, Joensuu H, Nordman E. Imaging of head and neck tumors with positron emission tomography and $\left[{ }^{11} \mathrm{C}\right]$ methionine. Int J Radiat Oncol Biol Phys. 1994;30:1195-1199.

71. Geets X, Daisne JF, Gregoire V, Hamoir M, Lonneux M. Role of ${ }^{11} \mathrm{C}$-methionine positron emission tomography for the delineation of the tumor volume in pharyngo-laryngeal squamous cell carcinoma: comparison with FDG-PET and CT. Radiother Oncol. 2004;71:267-273.

72. Sun A, Sorensen J, Karlsson M, et al. $1-\left[{ }^{11} \mathrm{C}\right]$-acetate PET imaging in head and neck cancer: a comparison with ${ }^{18}$ F-FDG-PET-implications for staging and radiotherapy planning. Eur J Nucl Med Mol Imaging. 2007;34:651-657.

73. Cai W, Chen K, He L, Cao Q, Koong A, Chen X. Quantitative PET of EGFR expression in xenograft-bearing mice using ${ }^{64} \mathrm{Cu}$-labeled cetuximab, a chimeric anti-EGFR monoclonal antibody. Eur J Nucl Med Mol Imaging. 2007;34: $850-858$.

74. Wang JQ, Gao M, Miller KD, Sledge GW, Zheng QH. Synthesis of $\left[{ }^{11} \mathrm{C}\right]$ Iressa as a new potential PET cancer imaging agent for epidermal growth factor receptor tyrosine kinase. Bioorg Med Chem Lett. 2006;16:4102-4106.

75. Are You HD Ready? [product information, HD-PET]. Knoxville, TN: Siemens; 2007.

76. Judenhofer MS, Wehrl HF, Newport DF, et al. Simultaneous PET-MRI: a new approach for functional and morphological imaging. Nat Med. 2008;14: 459-465. 